\title{
Hyperpigmentation as a peculiar presentation of mycosis fungoides*
}

\author{
Ying-Yi Lu' ${ }^{1,2,3}$ \\ Chun-Ching $\mathrm{Lu}^{6}$
}

\author{
Chieh-Hsin $\mathrm{Wu}^{2,4,5}$ \\ Chien-Hui Hong ${ }^{1,7}$
}

DOI: http://dx.doi.org/10.1590/abd1806-4841.20175544

\begin{abstract}
Hyperpigmented mycosis fungoides is an extremely rare subtype of mycosis fungoides. It presents as multiple pigmented macules and patches without poikilodermatous changes and characterized by a CD8+ phenotype on immunohistochemistry. This report describes a typical case of hyperpigmented mycosis fungoides in a 62-year-old woman, who presented with a 7-year history of multiple hyperpigmented macules and patches on the trunk and right leg with progression over this half a year. Histology and immunohistochemical staining of skin samples confirmed the diagnosis of mycosis fungoides. She received psoralen plus ultraviolet A (PUVA) therapy. After an 8-week treatment, the erythematous changes cleared without recurrence during a 6-month follow-up period. An intractable hyperpigmented patch should raise the clinical suspicion of mycosis fungoides with sequential skin biopsy.
\end{abstract}

Keywords: Antigens, CD8; Hyperpigmentation; Mycosis fungoides

\section{INTRODUCTION}

Mycosis fungoides (MF) is the most common form of cutaneous T cell lymphoma. Hyperpigmented mycosis fungoides is among the rarest variant of MF, manifested as hyperpigmented macules or patches without poikilodermatous changes such as epidermal atrophy and telangiectasias. ${ }^{1}$ It is characterized by a predominantly $\mathrm{CD} 8+$ phenotype. Additionally, it usually affects younger patients and is more predominant in dark-skinned complexions. Most important of all, it has a favorable prognosis. So far, only eight cases have been reported. Herein, we describe a patient with several hyperpigmented macules and patches on the trunk and right leg for 7 years previously. A diagnosis of hyperpigmented MF was established on histopathology. After an 8-week treatment of PUVA, the erythematous changes regressed.

\section{CASE REPORT}

A 62-year-old otherwise healthy Taiwanese woman presented with a 7-year history of nonpruritic, multiple black-colored hyperpigmented patches without scaling, atrophy or telangiecta-

sias on her trunk and right leg. Similar hyperpigmented patches developed and progressed on her chest and flank over half a year (Figure 1). Some of the pigmented patches had become surrounded with erythema two weeks prior to presentation (Figure 1). She denied use of special medications and she had no known history of dermatological diseases. There was no fever, body weight loss, or night sweating, neither was palpable lymphadenopathy nor hepatosplenomegaly present upon physical examination. Because of intractable eruptions, she underwent a skin biopsy. The histology of lesional skin from a hyperpigmented patch and surrounding erythema both showed prominent epidermotropism and atypical lymphocytes (small to medium-sized hyperchromatic indented nuclei) aligning along the basal layer and perivascular space with dermal melanophages (Figure 2). Immunochemistry showed atypical lymphocytes positive for $\mathrm{CD} 3$ and $\mathrm{CD} 8$ but negative for $\mathrm{CD} 4$, CD7, CD20 and S100. Her blood count, blood biochemistry panel including lactate dehydrogenase level, tumor markers, coagulation profiles, T-cell subset analysis, serum protein electrophoresis, chest X-ray, abdominal ultrasonography, bone marrow aspiration and bi-

Study submitted on 05.01 .2016

Approved by the Advisory Board and accepted for publication on 16.04.2016

* Work performed at the Department of Dermatology, Kaohsiung Veterans General Hospital - Kaohsiung, Taiwan.

Financial support: None

Conflict of interest: None

\author{
Department of Dermatology, Kaohsiung Veterans General Hospital- Kaohsiung, Taiwan. \\ Graduate Institute of Medicine, College of Medicine, Kaohsiung Medical University- Kaohsiung, Taiwan. \\ Department of Cosmetic Applications and Management, Yuh-Ing Junior College of Health Care \& Management - Kaohsiung, Taiwan \\ Division of Neurosurgery, Department of Surgery, Kaohsiung Medical University Hospital- Kaohsiung, Taiwan. \\ Department of Surgery, School of Medicine, College of Medicine, Kaohsiung Medical University- Kaohsiung, Taiwan. \\ Department of Orthopedics, Taipei Veterans General Hospital - Taipei, Taiwan. \\ Department of Dermatology, National Yang-Ming University, College of Medicine - Taipei, Taiwan.
}

(C2017 by Anais Brasileiros de Dermatologia 
opsy were all unremarkable. Fluorodeoxyglucose positron emission tomography showed no active signs of visceral involvement. Based on the pathology, she was diagnosed as stage I CD8+ hyperpigmented mycosis fungoides. She then received psoralen plus ultraviolet $\mathrm{A}$ therapy with oral 8-methoxypsoralen three times a week along with a potent topical corticosteroid agent (clobetasol propionate). After 8 weeks of treatment, the erythematous lesions regressed with no development of new lesions, and the involved pigmented area had reduced from $10 \%$ to $6 \%$ of the total body surface area (Figure 3 ).
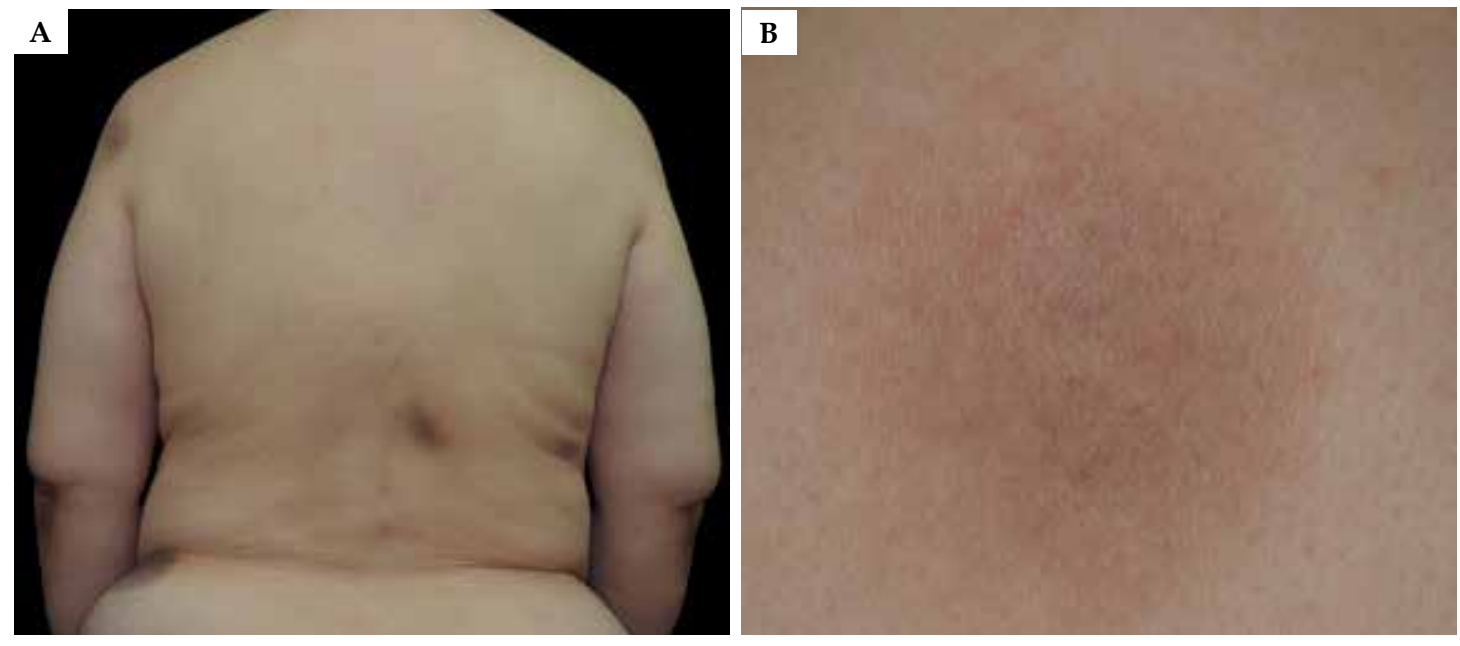

Figure 1:

A. Multiple black-colored, hyperpigmented patches without scaling, atrophy and telangiectasias on the trunk. B. Patches with surrounding erythema
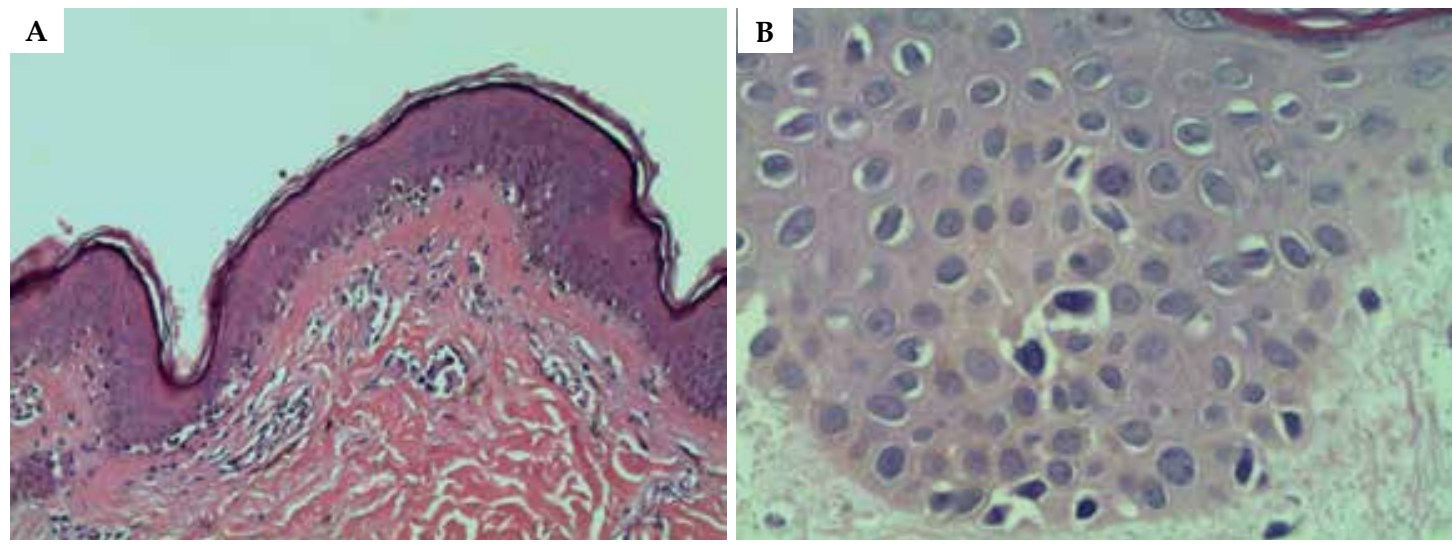

Figure 2: A. Histopathology showing epidermotropism, atypical lymphocytes aligning along the basal layer and perivascular space with melanophages in the dermis (Hematoxylin \& eosin, X200). B. High power view demonstrating atypical small to medium-sized hyperchromatic lymphocytes (Hematoxylin \& eosin, X400)
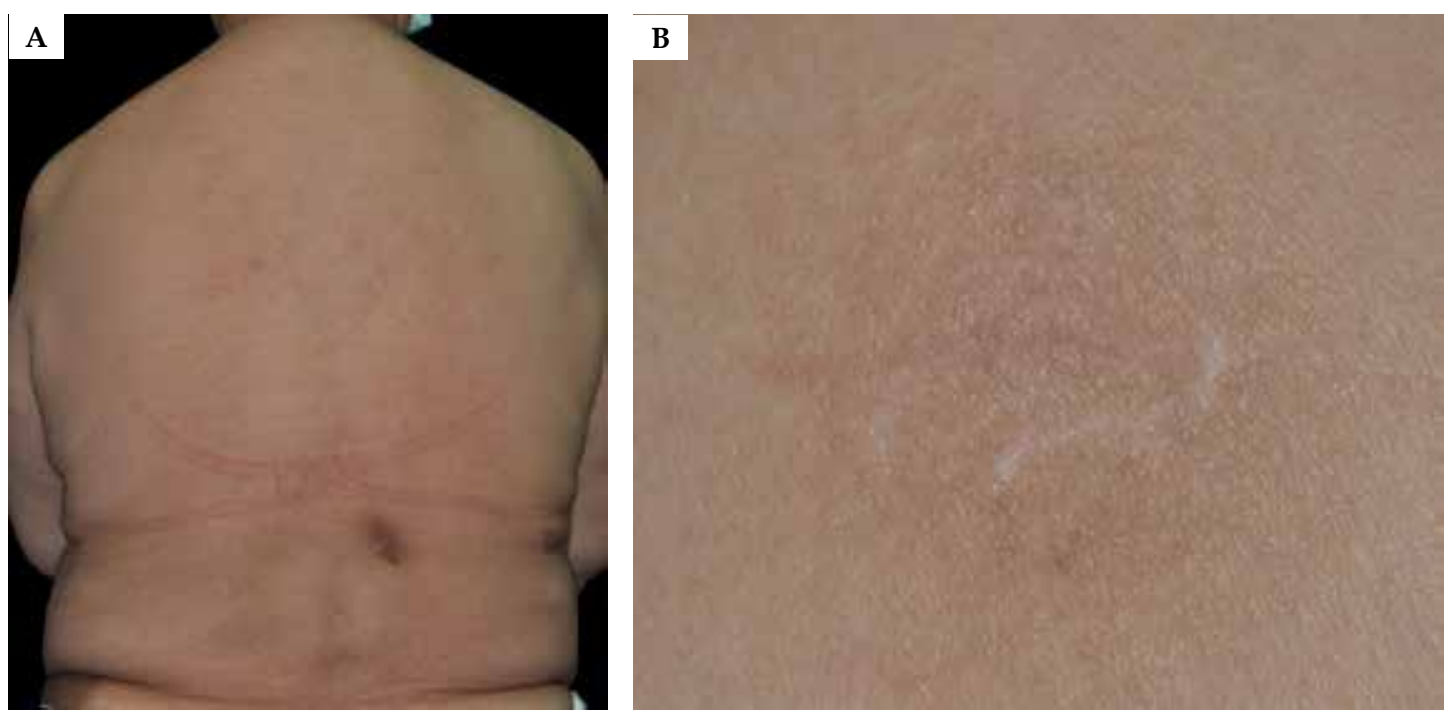

Figure 3:

A. Regression of hyperpigmented patches. B. The patch with surrounding diminished erythema after 8-week treatment of PUVA 


\section{DISCUSSION}

Mycosis fungoides is the most common type of cutaneous T-cell lymphoma, and classic-type MF evolves clinically through patch, plaque and tumor stages. MF is characterized by a clonal proliferation of CD4+ T cells or, less often, by a CD8+ or CD4- CD8phenotype. Atypical clinical variants include angiocentric, bullous, erythrodermic, follicular, granulomatous, hypopigmented, hyperpigmented, ichthyosiform, mucinous, palmoplantar, pigmented purpura-like, poikilodermatous, pustular, syringotropic, unilesional and verrucous/ hyperkeratotic subtypes. ${ }^{2-5}$

Hyperpigmented MF is the rarest form, with only eight cases being reported to date ${ }^{1}$. Hyperpigmented MF usually affects individuals with a dark complexion and a younger age (less than 35 years old) than patients with classic MF. It presents as hyperpigmented patches and plaques without evidence of poikilodermatous changes. ${ }^{1}$ The histology is characterized by interface changes with melanophages on hematoxylin-eosin staining in addition to epidermotropism and dermal infiltration of lymphocytes, the classical findings of MF, and immunohistochemically by a predominantly CD8+ and less often, by a CD4- CD8- phenotype. ${ }^{3}$ Ultrastructural studies show the presence of giant melanin granules in atypical lymphocytes, macrophages, keratinocytes and Langerhans' cells of the epidermis. ${ }^{4}$ The pathogenesis remains unknown. The peculiar clinical appearance of hyperpigmentation may be the result of interface changes and melanophages, because suppressor or cytotoxic CD8+ $\mathrm{T}$ cells affect melanocytes and basal keratinocytes ${ }^{3}$. In addition, the role of mast cell and stem cell factor in hyperpigmented MF has been proposed, which may stimulate the production of melanin by melanocytes. ${ }^{6,7}$ Hyperpigmented MF may appear solely, or concurrently with other pigmentary lesions of MF such as poikilodermatous, hypopigmented and pigmented purpura-like types ${ }^{3}$. In addition, it has an indolent course, similar to classic early-stage MF with a more favorable prognosis, whereas classic MF with a CD8+ phenotype shows a more aggressive behavior. ${ }^{2,3}$

\section{REFERENCES}

1. Erbil H, Sezer E, Koseoglu D, Filiz N, Kurumlu Z, Bülent Taştan H, et al. Hyperpigmented mycosis fungoides: a case report. J Eur Acad Dermatol Venereol. 2007;21:982-3.

2. Lee JS, Yun SJ, Lee JB, Kim SJ, Won YH, Lee SC. A case of hyperpigmented mycosis fungoides: a rare variant. J Eur Acad Dermatol Venereol. 2007;21:983-5

3. Pavlovsky L, Mimouni D, Amitay-Laish I, Feinmesser M, David M, Hodak E. Hyperpigmented mycosis fungoides: An unusual variant of cutaneous T-cell lymphoma with a frequent CD8 + phenotype. J Am Acad Dermatol. 2012;67:69-75.

4. Kazakov DV, Burg G, Kempf W. Clinicopathological spectrum of mycosis fungoides. J Eur Acad Dermatol Venereol. 2004;18:397-415.

5. Namazi MR. Ecchymosis-like hyperpigmented mycosis fungoides. Ann Saud Med. 2008;28:228-9.

6. Yamamoto T, Katayama I, Nishioka K. Increased serum level of stem cell factor in association with disease progression of hyperpigmented mycosis fungoides. $\mathrm{Br} J$ Dermatol. 1999;140:765-6.
There are many differential diagnoses for acquired circumscribed hyperpigmented patches, including postinflammatory hyperpigmentation, erythema dyschromicum perstans, cutaneous amyloidosis, atrophoderma of Pasini-Pierini, parakeratosis variegate, pigmented purpura-like MF, poikiloderma vasculare atrophicans, idiopathic eruptive macular hyperpigmentation, drug eruptions and contact dermatitis. ${ }^{1,3}$ The histopathologic diagnosis of early MF can be extremely difficult. Multiple biopsies over several months may be required to confirm the clinical suspicion, and in most cases the definite diagnosis must be established by comprehensive clinicopathological correlation.

The treatment of hyperpigmented MF depends on the clinical stages, which is similar to classic MF. Early aggressive therapy doses not improve survival. Photochemotherapy is the standard therapy for the early stage of MF. ${ }^{8}$ Besides PUVA, topical corticosteroid agents, topical chemotherapy, interferon alpha-2a and oral retinoids can be used. ${ }^{8}$ Combination therapies have become more popular recently. Systemic chemotherapy and new immunologic agents are usually saved for the advanced stages with nodal or visceral involvement.

In summary, there are differences between hyperpigmented MF and classic MF. Hyperpigmented MF usually affects younger patients than classic MF, and it tends to affect dark-skinned individuals. In addition, its immunophenotype shows CD8+ predominance whereas the classic type shows CD4+ predominance, and it has a better prognosis than the classic type. ${ }^{1-3}$ From a clinical point of view, mycosis fungoides should be added to the differential diagnoses in any case of refractory hyperpigmented dermatosis. A skin biopsy should be performed appropriately and sequentially in patients with persistent hyperpigmented patches to rule out this possibility. ${ }^{1} \square$
7. Yamamoto T, Katayama I, Nishioka K. Role of mast cell and stem cell factor in hyperpigmented mycosis fungoides. Blood. 1997;90:1338-40.

8. Mataix J, Bañuls J, Lucas A, Belinchón I, Betlloch I. Poikilodermatous mycosis fungoides. Int J Dermatol. 2007;46:950-1.

MAILING ADDRESS:

Ying-Yi Lu

$N^{\circ} .386$ Ta-Chung 1st Rd.

Kaohsiung City

81362, Taiwan

E-mail: actinpactinp@yahoo.com.tw,myosinpp@yahoo.com.tw

How to cite this article: $\mathrm{Lu} \mathrm{YY}, \mathrm{Wu} \mathrm{CH}, \mathrm{Lu} \mathrm{CC}$, Hong $\mathrm{CH}$. Pneumocystis jirovecii pneumonia induced by low-dose methotrexate in a patient with chronic urticaria. An Bras Dermatol. 2017;92(5 Suppl 1): 92-4. 